\title{
PROMOÇÃO E EDUCAÇÃO EM SAÚDE NOS NÚCLEOS DE APOIO À SAÚDE DA FAMÍLIA (NASF) DE CAMPINA GRANDE E JOÃO PESSOA/PB
}

\author{
Camila Rachel Lira Silva ${ }^{1}$ \\ Alexandra Bonifácio Xavier ${ }^{2}$ \\ Sandra Amélia Sampaio Silveira ${ }^{3}$ \\ Kathleen Elane Leal Vasconcelos ${ }^{4}$
}

\begin{abstract}
Resumo:O Núcleo de Apoio à Saúde da Família (NASF) foi implantado em 2008 com o objetivo de ampliar a abrangência e o escopo da Atenção Primária à Saúde (APS). Para tanto, deve estabelecer relação de apoio às equipes de Saúde da Família. Este artigo busca analisar as concepções em torno da Promoção da Saúde (PS) e Educação em Saúde dos/as profissionais dos NASFs de Campina Grande e João Pessoa/PB, a partir de uma pesquisa de campo realizada juntos aos mesmos. Os resultados indicam que a maioria dos profissionais tende a associar a concepção de Promoção da Saúde com prevenção de doenças e a Educação em Saúde com a realização de grupos.

Palavras-chave: Educação em Saúde; Promoção da Saúde; Núcleo de Apoio à Saúde da Família.
\end{abstract}

\footnotetext{
${ }^{1}$ Serviço Social/Universidade Estadual da Paraíba, Brasil. E-mail: miila-lira@hotmail.com.

${ }^{2}$ Serviço Social/Universidade Estadual da Paraíba, Brasil. E-mail: maurithiuseale@hotmail.com.

${ }^{3}$ Serviço Social/Universidade Estadual da Paraíba, Brasil. E-mail: samelias2@yahoo.com.br.

${ }^{4}$ Serviço Social/Universidade Estadual da Paraíba, Brasil. E-mail: prof.kathleen.uepb@gmail.com.
} 